\title{
Jenseits der Klinik: Konzeptionelle Überlegungen zum Ethiktransfer in dezentralen Einrichtungen des Gesundheitswesens am Beispiel der BruderhausDiakonie Reutlingen
}

\author{
Christiane Burmeister • Ariane Iller · Robert Ranisch • Cordula Brand • \\ Tobias Staib • Uta Müller
}

Eingegangen: 22. Dezember 2020 / Angenommen: 1. April 2021 / Online publiziert: 27. Mai 2021

(C) Der/die Autor(en) 2021

Zusammenfassung Unser Beitrag stellt ein interaktives Ethik-Konzept vor, das in Zusammenarbeit der BruderhausDiakonie Reutlingen und der Universität Tübingen entwickelt wurde, um den Eigenheiten und Bedarfen einer komplexen Organisationsstruktur gerecht zu werden, die mehrere Geschäftsfelder und Standorte unter sich vereint. Wir skizzieren die Grundzüge des interaktiven Nijmegener Modells, in dem die Kooperation eines auf Leitungsebene angesiedelten Komitees und situationsbezogener Fallbesprechungen ein fruchtbares Zusammenspiel zweier unverzichtbarer Reflexionsweisen bewirken soll (,Top-Down“/,Bottom-Up“). Wir zeigen auf, welche Herausforderungen sich bei der Implementierung dieses Modells in die konkrete Aufbauorganisation der BruderhausDiakonie ergaben, und mit welchen konzeptionellen oder ,implementationstechnischen“ Mitteln ihnen begegnet wurde. Im Zentrum steht dabei die Erweiterung des Nijmegener Modells um ein Verbindungselement, welches die Zusammenarbeit zwischen zentralem Ausschuss und dezentralen Fallbesprechungen koordiniert und das interaktive Moment des Modells erst ermöglicht.

Christiane Burmeister, M.A. ( $₫)$

Internationales Zentrum für Ethik in den Wissenschaften (IZEW) Tübingen, Universität Tübingen, Wilhelmstraße 19, 72074 Tübingen, Deutschland

E-Mail: christiane.burmeister@uni-tuebingen.de

Ariane Iller, M.A. · Dr. Tobias Staib

BruderhausDiakonie Reutlingen, Reutlingen, Deutschland

Prof. Dr. Robert Ranisch

Fakultät für Gesundheitswissenschaften Brandenburg, Universität Potsdam, Potsdam, Deutschland

Dr. Cordula Brand · Dr. Uta Müller

Internationales Zentrum für Ethik in den Wissenschaften, Universität Tübingen, Tübingen, Deutschland 
Schlüsselwörter Organisationsethik · Implementierung $\cdot$ Ethikkomitee ·

Fallbesprechung · Praxisbericht · Nimwegener Modell · Ethische Fallbesprechung

\title{
Beyond the clinic. Conceptual considerations on transferring ethics to decentralized health care facilities using the example of the BruderhausDiakonie Reutlingen
}

\begin{abstract}
Definition of the problem Medical and nursing care often takes place within complex organizational structures that comprise numerous facilities at numerous locations. We introduce an interactive ethical concept, designed in cooperation with the diaconal foundation BruderhausDiakonie Reutlingen and the International Centre for Ethics in Science, University of Tübingen, to address the particular needs of such organizations.

Arguments Therefore we portray the interactive Nijmegen Model which combines an ethics committee located at the management level and situational ethical case deliberations on the ward in order to bring together two indispensable ways of ethical reflection in organizations: "top-down"/"bottom-up". We illustrate the challenges of implementing the said model into the organizational construction of the BruderhausDiakonie and how to address these by means of conception and implementation.

Conclusion Considering the implementational challenges that we encountered, the Nijmegen Model needs to be enhanced by introducing a link between the committee and the case deliberations. We delineate the basic elements and the requirements for such a position while reporting our initial practical experience with this model.
\end{abstract}

Keywords Organizational ethics · Implementation · Ethics committee · Case deliberations $\cdot$ Nijmegen interactive model $\cdot$ Practice report $\cdot$ Moral case deliberation

\section{Einleitung}

Der vorliegende Bericht beruht auf den Ergebnissen einer transdisziplinären Zusammenarbeit zwischen der gemeinnützigen Stiftungsorganisation BruderhausDiakonie Reutlingen, Trägerin von Einrichtungen verschiedener gesundheitlicher wie sozialer Assistenzleistungen, und dem Internationalen Zentrum für Ethik in den Wissenschaften (IZEW) der Universität Tübingen. Diese Zusammenarbeit gründete in dem Bestreben der BruderhausDiakonie, die Qualität ethischer Entscheidungsfindung zu verbessern und normative Diskursstrukturen auf verschiedenen Ebenen zu verankern. Zu diesem Zweck wurden im Zeitraum von 2016 bis 2018 konzeptionelle Beratungssitzungen in Hinblick auf ein geplantes, die gesamte Organisation umfassendes Ethikmanagement durchgeführt. In zweiter Linie erstreckte sich die 
Zusammenarbeit auf verschiedene Weiterbildungsformate ${ }^{1} \mathrm{zu}$ berufs- und organisationsethischen Themen. Wir konzentrieren uns im Folgenden auf die relevanten konzeptionellen Überlegungen, mit denen das ideelle Konstrukt eines organisationsethischen Modells in eine real existierende Aufbauorganisation übertragen wurde. Die Herausforderungen, die uns durch die besondere Struktur dieser komplexen Organisation begegnet sind, warfen zugleich Licht auf eine mögliche Schwachstelle des gewählten ethischen Modells: die fehlende Verknüpfung der einzelnen Organe. Unsere vorgeschlagene Weiterentwicklung soll diese Schwachstelle beheben und die gewünschte interaktive Funktionsweise erleichtern. Unsere Überlegungen können damit erste Anregungen für Organisationen mit vergleichbaren Strukturvoraussetzungen bieten, die vor ähnlichen Herausforderungen stehen, wie im Folgenden beschrieben.

\section{Die BruderhausDiakonie Reutlingen ${ }^{2}$}

Als dezentrale Stiftung mit christlichem Profil und über 5000 Mitarbeiter*innen aus den Bereichen Altenhilfe, Behindertenhilfe, Jugendhilfe, Sozialpsychiatrie, Arbeit und berufliche Bildung unterhält die BruderhausDiakonie Einrichtungen in 45 Standorten Baden-Württembergs, verteilt von Südbaden bis Ostwürttemberg. Damit gehört sie zu den größten Anbietern sozialer, pflegerischer und medizinisch-therapeutischer Assistenzleistungen in der Region Reutlingen. Die medizinisch-therapeutischen und pflegerischen Tätigkeiten zentrieren sich vorrangig in den ambulanten wie stationären Diensten der Altenhilfe, Behindertenhilfe und Sozialpsychiatrie. Die Zusammenarbeit mit nicht-medizinischen Fachdisziplinen, etwa pädagogischer Ausrichtung oder mit Berufsgruppen der Sozialen Arbeit, gehört für die Mitarbeitenden der BruderhausDiakonie zum beruflichen Alltagsgeschehen. Als größte Bereiche sind die Geschäftsfelder Altenhilfe und Sozialpsychiatrie zu nennen. Entsprechend betreffen die größten ethischen Unsicherheiten Behandlungsfragen am Lebensende oder den Umgang mit herausfordernden/zentrale Versorgungsleistungen verweigernden Patient*innen und Klient*innen.

Bis zum Zeitpunkt der Zusammenarbeit mit dem IZEW existierten in der Organisation einzelne, unstrukturierte Aktivitäten im Bereich „Ethik“. So begannen im Jahr 2014 unabhängig voneinander kleinere Arbeitskreise aus der Mitarbeiterschaft mit der Entwicklung von Positionspapieren zu konkreten ethischen Themen aus der Fachpraxis (z. B. „Lebensqualität“”, „Umgang mit Sexualität“, „Akutsituationen mit ethischem Entscheidungsdruck“), während in Leitungskonferenzen über die ethische Profilierung der Gesamtorganisation diskutiert wurde. Auch gab es die Möglichkeit, einen in ethischer Fallbesprechung fortgebildeten Pfarrer zu Rate zu ziehen, der aufgrund seines persönlichen Engagements, aber auch aufgrund der Singularität seiner formalen Funktion als Leiter der Abteilung ,Theologie und Seelsorge“ ein hohes $\mathrm{Ma}$ an Bekanntheit und Vertrauen in der Gesamtorganisation genoss. Dieses Ver-

\footnotetext{
1 Hierbei handelte es sich um Fortbildungskurse für die Führungskräfte der mittleren Ebene, Inhouse-Workshops für Führungskräfte der höheren Leitungsebene und berufsbegleitende CAS-Seminare (,Ethik in Organisationen“), u. a. für die Ethikbeauftragte und ein Vorstandsmitglied der BruderhausDiakonie.

2 https://www.bruderhausdiakonie.de/unternehmen. Zugegriffen: 12. April 2021.
} 
trauen kompensierte zwar ein Stück weit das Fehlen von unterstützenden Strukturen seiner Arbeit, einen systematischen Umgang mit ethischen Konfliktfällen ersetzte diese auf einer Person basierende Struktur allerdings nicht. Neben dem Problem der fehlenden Trennung von theologischer Seelsorge und ethischer Beratung blieb die Reichweite einer Einzelperson in einem derart großen Unternehmen zu gering. Nicht zuletzt besteht mit dem ,singulären Beratermodell“ wenig Hoffnung auf ein Wachstum an ethischer Kompetenz in der Mitarbeiterschaft, da ethische ,Aspekte an den [...] Ethiker als Spezialisten der Institution delegiert werden“ (Vollmann 2002, S. 279). Die Notwendigkeit systematischer und koordinierter Ethikarbeit erschien darum offensichtlich.

Im Jahr 2014 wurde schließlich ein Projekt zur Implementierung strukturierter Ethikarbeit in der BruderhausDiakonie beschlossen, dessen Planungsteam die beiden Leiter der Stiftungsmanagements „Unternehmensentwicklung“ und ,Theologie und Seelsorge“" sowie die Leiterin des Bereichs Personalentwicklung darstellten. Um den Bedarf nach ethischer Unterstützung in detaillierterer Form zu erheben, wurden strukturierte Interviews mit einem alle Hierarchieebenen, Geschäftsfelder und Regionen repräsentierenden Anteil des Personals durchgeführt, nach denen im Wesentlichen folgende Bedürfnisse herausgestellt werden konnten:

1. ethische Orientierung strukturierter Unterstützung bei ethisch herausfordernden Alltagssituationen

2. Befähigung der Mitarbeitenden und Führungskräfte zum Umgang mit ethischen Fragestellungen

3. Klärung von Haltungen des Trägers zu ethischen Fragestellungen (bspw. in Positionspapieren)

4. Kommunikation des diakonischen Profils und Leitbilds (BruderhausDiakonie 2020), insbesondere gegenüber Patient*innen, Klient*innen und Angehörigen

Mit diesen Zielstellungen und einem in Leitungskonferenzen verabschiedeten Budget für die Realisierung eines umfassenden Konzepts, wurde schließlich im Jahr 2016 die Zusammenarbeit mit dem IZEW im nahegelegenen Tübingen aufgenommen.

\section{Konzeptionelle Vorüberlegungen}

Inhalt der ersten Beratungssitzungen war es, ein Grundkonzept der Ethikberatung zu ermitteln, das den Zielsetzungen gemäß o.g. Bedarfsfeststellung entsprach und zugleich mit den vorhandenen Strukturbedingungen der BruderhausDiakonie vereinbar war. Festgehalten werden konnte, dass sowohl die ethische Reflexion individueller Fragestellungen aus der Patient*innen-/Klient*inneninteraktion als auch eine Ethikarbeit mit Blick auf und Geltungsanspruch für die Gesamtorganisation etabliert werden sollte. Aus den Zielsetzungen ging zudem hervor, dass das gesuchte Modell sowohl zur Entwicklung normativer Richtlinien beitragen, also „Compliance“basierte Elemente enthalten sollte (Punkte 1 und 3), als auch die Stärkung der Eigenverantwortlichkeit fördern, also „Integrity“-orientierte Steuerungsmechanismen enthalten sollte (Punkt 2). 
Darüber hinaus musste das gesuchte Modell mit bestimmten Rahmenbedingungen der Organisation kompatibel sein. Hierzu gehörte die Einsatzfähigkeit für verschiedene professionelle Handlungsfelder von der Sozialpsychiatrie bis zur Behindertenhilfe (auch, wenn der augenscheinlichste Bedarf für ethische Reflexionsarbeit in den Bereichen Altenhilfe und Sozialpsychiatrie herrschte). Ferner erschien eine ausschließlich in der Hauptverwaltung Reutlingen lokalisierte „Anlaufstelle“ für ethische Fragen aufgrund der überregionalen Verteilung der Standorte nicht ausreichend. Eine situationsnahe Verfügbarkeit von Ethikberatung vor Ort wurde als wesentliches Merkmal identifiziert. Nicht zuletzt galt es, die Matrixstruktur des Unternehmens und damit das Vorhandensein verschiedener, gleichberechtigter Hierarchielinien zu berücksichtigen. All dies machte deutlich, dass das zu implementierende Modell auf besondere Weise in das Organigramm der BruderhausDiakonie eingepasst werden musste, um sich in aktive Ethikarbeit umsetzen zu können.

\section{Vorlage: Das Nijmegener Modell}

Das „Nijmegener Modell“ der Medizinethiker Steinkamp und Gordijn (2010) bot sich insofern als erste Vorlage an, als es nicht nur die geeigneten Instrumente der ethischen Reflexion enthielt, sondern auch implementationsbezogene Überlegungen einbezog. Wie die niederländischen Medizinethiker betonen, hängt ,es von der Art und Weise der Implementierung [ab], ob die durch ethisches Denken ermöglichte Dynamik der Veränderung auch tatsächlich zum Tragen kommt“ (Steinkamp und Gordijn 2010, S. 143).

Wenngleich es am Organisationstypus Klinik orientiert ist, lässt sich das Modell in seinen Grundzügen auch auf außerklinische Organisationen anwenden, insofern hier wie dort zwei institutionelle Ebenen der Ethikberatung unterschieden werden können: Die erste bezieht sich auf die Organisation als Ganzes, die zweite adressiert die fallbezogene professionelle Tätigkeit (vgl. Vollmann 2008, S. 117). Mit beiden Perspektiven geht eine grundlegend verschiedene Art der Auseinandersetzung mit ethischen Fragen einher. Während in Perspektive der Gesamtorganisation allgemeinere Orientierungsschriften ausgearbeitet werden, aus denen im Einzelfall konkretere Aussagen deduziert werden müssen (,,Top-Down“), knüpft die „Ethik am Krankenbett“" an der konkreten Situationserfahrung an, von der ausgehend sie Prinzipien kontextualisiert und verhandelt (,Bottom-Up“) (vgl. Steinkamp und Gordijn 2010, S. 127 ff.).

Um diese beiden „Fließrichtungen der ethischen Reflexion“"weder zu vermischen, noch gegeneinander auszuspielen, werden ihnen von Steinkamp und Gordijn zwei unabhängig arbeitende aber sich wechselseitig unterstützende Instrumente zugewiesen:

1. ein multidisziplinär und repräsentativ besetztes, mit ethischen Fragen und Leitlinien der Gesamtorganisation befasstes Ethikkomitee auf der einen Seite,

2. die den Einzelfall auf Station reflektierenden ethischen Fallbesprechungen auf der anderen Seite.

Von Bedeutung ist nun, dass das Nijmegener Modell diese beiden Instrumente nicht lediglich als getrennte Arbeitsaufträge für ein- und dasselbe ausführende Gre- 
mium versteht, sondern in der Verantwortung verschiedener Organe sieht, welche zusätzlich auf verschiedenen Organisationsebenen zu verankern sind.

Damit vertreten die Autoren die ausdrückliche Gegenposition zu jenem Modell, nach dem Einzelfallberatungen zu den Hauptaufgaben eines Klinischen Ethikkomitees zählen (vgl. auch Vollmann 2017, S. 182). Zwar räumen Gordijn und Steinkamp der disziplinenübergreifenden Perspektivenvielfalt innerhalb eines Ethikkomitees auch für die Beratung in einzelnen Handlungssituationen einen Vorteil ein. Allerdings ginge mit der zwangsläufigen Distanz der Mitglieder zum jeweiligen Fall ein Zugang von ,besonderer Qualität“ verloren, der sich nur durch das konkrete Verantwortungsgefühl eines behandelnden Teams, die konkrete professionelle Beziehung zum Patienten, ergeben könne (Steinkamp und Gordijn 2010, S. 143).

Ein ernst zu nehmender Einwand gegen diese Position ist freilich, dass gerade die in der Extraposition der Urteilenden gründende Distanz jene intersubjektive Haltung ermöglicht, welche für die ethische Beurteilung eines Konfliktfalls von entscheidender Bedeutung ist. Hier ist insbesondere zu berücksichtigen, dass es im Fall der BruderhausDiakonie nicht nur um medizinisch-pflegerische, sondern zu einem Großteil um Entscheidungssituationen in der Sozialen Arbeit geht, für die die ,sozialanwaltliche Parteinahme zugunsten des Klienten“ (Maaser 2013, S. 7) zum professionellen Selbstverständnis gehört (vgl. auch Thiersch 2015, S. 1061). Gleichwohl disqualifiert dieser Zugang die Verantwortlichen nicht für die Teilnahme am ethischen Entscheidungsfindungsprozess. Er verweist vielmehr auf die Notwendigkeit verfahrensethischer Rahmenbedingungen - allen voran eine in ethischen Grundkompetenzen geschulte Moderation -, damit sich alle beteiligten und betroffenen Perspektiven in einen fairen Deliberationsprozess begeben können. Genau hier knüpft schließlich die Überlegung an, dass das Prinzip der im verantwortlichen Team selbst durchgeführten Fallbesprechung am ehesten dazu geeignet ist, die Entwicklung ethischer Kompetenzen und einer Best Practice zu fördern (vgl. Steinkamp und Gordijn 2010, S. 143 f.).

Aus diesen Überlegungen ergibt sich nun folgende Aufgabenverteilung: Während die moderierten Fallbesprechungen vor Ort mithilfe eines verfahrensethisch gestalteten Gesprächsprotokolls ethisch-moralische Konfliktfälle ad hoc einer strukturierten Reflexion und Bearbeitung zuführen, trägt das Ethikkomitee für gute Rahmenbedingungen dieser Fallbesprechungen Sorge, berät das Direktorium in ethischen Fragen und fördert durch die Entwicklung von ethischen Empfehlungen sowie Fort- und Weiterbildungsinhalten die Auseinandersetzung mit ethischen Aspekten der professionellen Arbeit (vgl. Steinkamp und Gordijn 2010, S. 207). Um hinlänglich mit der Arbeit des jeweils anderen Gremiums verbunden zu bleiben, wird ein ,ständiger, auf Kernaufgaben und Leitungsverantwortung bezogener Austausch“ (Steinkamp und Gordijn 2010, S. 161) empfohlen.

\section{Die Erweiterung: Austausch von Komitee und Fallbesprechung koordinieren}

Bei der Übertragung dieses Modells in die konkrete Aufbauorganisation der BruderhausDiakonie fiel zunächst auf, dass es zwar problemlos möglich war, die beiden 
Organe des Modells auf den vorgesehenen Ebenen zu konzipieren. So wurde nach Vorlage des Interaktionsmodells ein repräsentativ besetzter, in der Hauptgeschäftsstelle bzw. den zentralen Dienstleistungen angesiedelter Ethikausschuss konzipiert und die Weiterbildung einer hinreichenden Anzahl an Ethikmoderator*innen aus der eigenen Mitarbeiterschaft für die Abdeckung aller Standorte projektiert.

Keineswegs selbstverständlich aber erschien bereits auf dem Papier das selbsttätige Zusammenwirken dieser beiden Instrumente durch den erwähnten ,ständigen Austausch“. Es fragte sich, wie ein solcher herzustellen und aufrechtzuerhalten sei, damit aus der Theorie eines dynamischen und flexiblen Interaktionsmodells nicht bloß zwei parallele Sprachrohre werden würden, die voneinander losgelöste Diskurse produzieren.

Eine solche Verzahnung wäre in einer zentral strukturierten Organisation die regelmäßige Berichterstattung von Ethikmoderator*innen an den Ausschuss, kombiniert mit einem ständigen Sitz in ebendiesem. Diese Möglichkeit ist jedoch vor dem Hintergrund der dezentralen Extension der Organisation und der fachlichen Diversität der BruderhausDiakonie unzureichend, da ein*e Ethikmoderator*in jeweils nur aus der Praxis des eigenen Standorts, also aus einer sehr begrenzten Anzahl an Geschäftsbereichen, wenn überhaupt: Einrichtungen, berichten könnte. Es ist jedoch gerade die elementare Idee eines organisationsbezogen ,denkenden“ Ethikausschusses, Probleme mit Strukturrelevanz zu identifizieren, weshalb er um einen repräsentativen Überblick bemüht sein muss.

Hier setzte die Idee einer verknüpfenden und vermittelnden Schaltstelle an, die dafür Sorge trägt, dass beide Organe - Ethikausschuss und Fallbesprechungen - in ihren jeweiligen Perspektiven aufeinander bezogen bleiben. Entworfen wurde eine zentrale Koordinationsstelle mit der Bezeichnung „Ethikbeauftragte*r“, deren Funktion im Wesentlichen darin bestehen sollte, die Arbeit der Moderator*innen sowie des Ausschusses zu koordinieren, füreinander aufzubereiten und Interaktionswege in fruchtbare Bahnen zu lenken. Dieses zusätzliche Steuerungselement machte aus dem dyadischen Modell Gordijns und Steinkamps ein triadisches Interaktionsmodell (Abb. 1).

Abb. 1 Das triadische Interaktionsmodell der BruderhausDiakonie, basierend auf Steinkamp und Gordijn (2010, S 151)

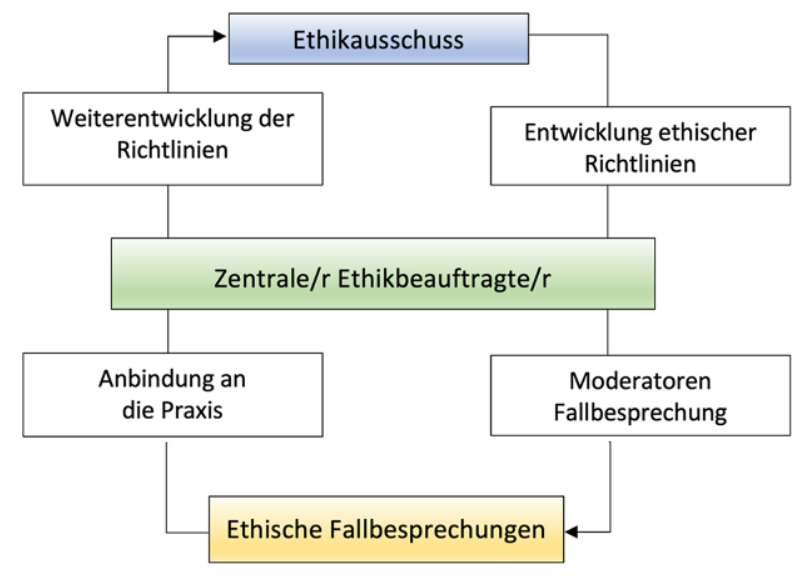


Wie diese Organe und ihr Zusammenwirken im Detail konzipiert sind und an welcher Stelle in der Organisationshierarchie sie installiert sind, soll im Folgenden mit besonderem Blick auf jene Fragen erläutert werden, die sich im Prozess der Implementierung gestellt haben.

\section{Der Ethikausschuss ${ }^{3}$}

Nach Vorlage des o.g. Grundentwurfs konzipierte das Planungsteam der BruderhausDiakonie einen vom Vorstand verabschiedeten, neunköpfigen Ethikausschuss mit dem grob zusammengefassten Aufgabenspektrum (1) Beratung des Vorstands, (2) Ausarbeitung und Unterstützung von Orientierungsschriften, (3) Unterstützung der Ethikmoderator*innen, sowie (4) (Weiter-)Entwicklung ethischer Fort- und Weiterbildung.

Mit der Zusammensetzung der Mitglieder sowie den Berufungsmodalitäten waren nun anspruchsvolle Fragen der Einordnung des Ausschusses in das horizontale Ebenensystem der BruderhausDiakonie verbunden. Einerseits galt es, ausreichend Gewicht und Wirkung zu erzielen, um in einer so großen Organisation einflussreich zu sein - ein Aspekt, der für die Zuordnung zur obersten Leitungsebene, dem Vorstand der BruderhausDiakonie, sprach. Ferner sprachen pragmatische Gründe wie die Einsparung von Kommunikationswegen für die Einbindung des Vorstandsvorsitzenden in den Ausschuss. Andererseits jedoch birgt eine solch enge Verbindung mit der „Befehlsgewalt“ die Gefahr, an Unabhängigkeit in der ethischen Argumentation einzubüßen - etwa durch hierarchiebedingte Effekte auf den möglichst freien und gleichberechtigten Diskurs oder eine strategische Themensetzung -, und von außen entsprechend wahrgenommen zu werden (Steinkamp und Gordijn 2010, S. 174). Für das Planungsteam der BruderhausDiakonie überwog allerdings die Idee der Praxiswirksamkeit, die mit einer engen Zusammenarbeit zwischen Vorstand und Ausschuss einherzugehen versprach. Darum wurde die feste Einbindung eines Mitglieds aus dem insgesamt dreiköpfigen Vorstand der BruderhausDiakonie in den Ethikausschuss beschlossen. Tatsächlich ist auch aus verfahrensethischer Sicht anzuerkennen, dass die administrativen Einblicke der Organisationsleitung oftmals zur Realität eines organisationsethisch relevanten Problems dazugehören und darum in die Fakten- und Kontextanalyse des Urteilsbildungsprozesses integriert werden sollten (Ranisch und Brand 2016). Entscheidend ist, dass strategische Überlegungen nicht die leitenden Einflüsse in der Diskussion werden.

Freilich zeigen sich diese nicht immer offen, auch wiegen die o.g. Einwände nach wie vor schwer. Darum wurde beschlossen, dem Ethikausschuss die Konzentration auf die reine ethische Argumentation zu gewähren und ihn von der Last strategischer Zwänge zu befreien. Entsprechend wurde seine Arbeit von der Inkraftsetzung der Empfehlungen getrennt. Die Ergebnisse der Ausschusssitzungen haben also ausdrücklich reinen Empfehlungscharakter. Ihre Umsetzung, bspw. als organisationsweit geltende Richt- oder Leitlinien, verbleibt in der Kompetenz des Organisationsvor-

\footnotetext{
${ }^{3}$ https://www.bruderhausdiakonie.de/unternehmen/theologie-und-diakonische-bildung/ethik. Zugegriffen: 20. April 2020.
} 
stands. Mittlerweile wurde zusätzlich konkretisiert, dass der Sitz im Ausschuss zwar vereinbar ist mit der Vorstandsmitgliedschaft, nicht aber mit dem Vorstandsvorsitz.

Die übrige Sitzverteilung beruhte auf den Prinzipien der Repräsentation und Partizipation. Im Fall der Matrixorganisation der BruderhausDiakonie hieß dies, mehrere Berufsgruppen und Führungslinien zu berücksichtigen. So wurden Sitze an eine*n Ethikmoderatoren*in vergeben, an eine Führungskraft aus der horizontalen Führungslinie, welche ein Angebotsspektrum sozialräumlich verortet, sowie an eine Geschäftsfeldleitung, welche die Qualität der Leistungserbringung und somit stärker fachliche Inhalte vertritt. Alle drei Personen sollten jeweils aus unterschiedlichen Geschäftsfeldern stammen. Hinzu kamen Sitze für die Leiter der Stiftungsmanagements „Unternehmensentwicklung“ sowie „Theologie und Seelsorge“. Der Vorsitz des Ethikausschusses wurde an ein Vorstandsmitglied gekoppelt, die Geschäftsführung des Ethikausschusses an das Amt der*des Zentralen Ethikbeauftragten. Diese*r ist mit Planung, Organisation und Nachbereitung der Sitzungen betraut sowie mit der Kommunikation bedeutsamer Entwicklungen an der „Basis“ des Ethikmanagements. Die Berichterstattung über die Arbeit der Ethikmoderator*innen oder kleinerer, mit ethischen Fragen (selbst)betrauter Arbeitsgruppen ist ein fixer Tagesordnungspunkt der Ausschusssitzungen. Zu guter Letzt wurden für die inhaltliche Qualität und den „Blick von außen“ zwei externe Fachleute aus der wissenschaftlichen Ethik besetzt. Die Berufung, welche durch den Vorstand erfolgt, wurde auf drei Jahre angelegt, der reguläre Sitzungsrhythmus halbtägig. Die Möglichkeit einer Bildung kleinerer Gruppen wurde als wünschenswerter Modus in der niedergeschriebenen Arbeitsweise festgehalten.

Ein vor kurzem abgeschlossenes Arbeitsergebnis aus dem Ethikausschuss ist bspw. eine Rahmenkonzeption, in der grundsätzliche Haltungsfragen der Organisation zum Thema „Sexualität im Betreuungskontext“ festgehalten und hinsichtlich verschiedener Dimensionen und Kontexte wie sexuelle Vielfalt und Identität, Beziehung und Partnerschaft, aber auch sexuelle Gewalt und Sexualassistenz konkretisiert wurden.

\section{Die Ethischen Fallbesprechungen (EFB)/Ethikmoderator*innen}

Um an allen regionalen Standorten und in allen Einrichtungen der BruderhausDiakonie die Möglichkeit einer schnell einsetzbaren EFB zur Verfügung zu stellen, wurden 13 interessierte Mitarbeiter*innen der BruderhausDiakonie zu Ethikmoderator*innen ausgebildet. Die vom diakonischen Dachverband verantwortete Schulung beinhaltete Basiswissen zu ethischen Schlüsselbegriffen, Einheiten zum Erkennen und Artikulieren ethischer Konfliktsituationen, Kenntnisse zu Modellen und Abläufen ethischer Fallbesprechungen und die Einübung von Moderationskompetenzen anhand des für die Praxis empfohlenen „Reflexionsmodells“ von Marianne Rabe (2017). Die Schulung umfasste insgesamt sechs Schulungstage, drei Fachkurse, drei kollegiale Beratungssitzungen und mehrere Übungsfallbesprechungen. Die auszubildenden Mitarbeiter*innen konnten aus den Bereichen Altenhilfe, Behindertenhilfe, Sozialpsychiatrische Hilfen und Jugendhilfe gewonnen werden. Da das Schulungs- 
programm weiter existiert, ist ein fortlaufendes Wachstum des Bestands an Ethikmoderator*innen theoretisch möglich.

Das Schulungskonzept sah vor, dass parallel zu den Moderator*innen auch deren jeweilige Führungskräfte geschult wurden, da diese als Multiplikatoren der Ethikarbeit und der EFB gewissermaßen die „Implementationsschrauben“ für das gesamte Instrument darstellen. Aus diesem Grund fanden entsprechende Begleitkurse für die Führungskräfte der Moderator*innen statt, deren Schwerpunkte auf organisationsethischen Fragen lagen. Mit diesem Hintergrund sollten die Führungskräfte in ihren Bereichen die wirkmächtigen „Komplizen“ des Instruments sein. An dieser Stelle sei die spätere Beobachtung vorweggenommen, dass eine enge Zusammenarbeit zwischen Ethikmoderator*innen und Führungskräften Spannungen produzierte. Gerade aufgrund des gemeinsamen Kompetenzerwerbs in ethischen Fragen ergaben sich anhaltende inhaltliche Deutungsstreitigkeiten. Kontrovers diskutiert wurde bspw., ob es sich bei einer Anfrage auf ethische Fallbesprechung tatsächlich um einen für dieses Instrument geeigneten, also ethischen Problemfall handelt. Für dieses Strukturproblem konnte jedoch unter Hinzuziehung der Beratung durch die Zentrale Ethikbeauftragte eine allgemeine Verfahrenslösung gefunden werden, indem der Auftragsformulierung eine Schlüsselfunktion zugewiesen wurde: Der von Führungskraft an Moderator*in gerichtete Auftrag sollte nicht im Entscheid über die Durchführung einer EFB bestehen, sondern in einer Antwort auf die Frage, ob es sich bei dem angetragenen Fall um einen ethischen Konflikt handelt. Dieser haarscharfe aber relevante Formulierungsunterschied dividierte die Kompetenzen von Moderator*in und Führungskraft wieder ansatzweise in argumentative Kompetenz auf der einen und administrative Autorität auf der anderen Seite, ein Spannungsfeld, das erfahrungsgemäß auf allen Ebenen der Organisation stets ausbalanciert werden muss.

Als ein wichtiger Standard wurde festgelegt, dass die Ethikmoderator*innen prinzipiell nicht für EFB in ihren eigenen Einrichtungen eingesetzt werden sollen. Zwar spricht die involvierte Position als Mitarbeiter*in/Kolleg*in eines Teams - wie weiter oben schon angemerkt - nicht gegen eine aktive Rolle in der EFB, sie ist jedoch grundsätzlich nicht mit der überparteilichen Rolle als Moderator*in vereinbar. Da nun zu bedenken war, dass die Moderator*innen in ihren eigenen Einrichtungen als kurzfristig und unkompliziert ansprechbare „Ethik-Expert*innen“ wahrgenommen werden und sich dadurch eine inoffizielle Praxis der Ethikberatung ,,auf kurzem Dienstweg“" ergeben könnte, wurde ein fest geregelter Ablauf der EFB entworfen, bei dem der*die zentrale Ethikbeauftragte eine entscheidende, verfahrenssichernde Rolle spielt.

Um in ihren Sonderrollen und in der dezentralen Weitläufigkeit der Organisation nicht zu „Einzelkämpfern“ (ZEKO 2006, S. A1705) zu werden, treffen sich die Ethikmoderator*innen zweimal im Jahr zu einem von der*dem Ethikbeauftragten verantworteten Reflexionstag. Dieser dient dem wechselseitigen Erfahrungsaustausch und der gemeinsamen Fortbildung zu unterschiedlichen Schwerpunkten, aber auch dem Überblick über die Bandbreite an Themen der bisher durchgeführten Fallbesprechungen. Eine erste Auswertung ergab, dass die häufigsten Fragestellungen die richtige Unterbringungsform von Klient*innen in Zusammenhang mit konfligierenden Interessen bzw. Bedürfnissen von zu Pflegenden/Klient*innen, Mitbewoh- 
ner*innen, Angehörigen und Pflegenden/Betreuenden betrafen. An zweiter Stelle stehen Unsicherheiten über das Ausmaß an Versorgung bzw. Fürsorge im Verhältnis zur Entscheidungsfreiheit der zu Versorgenden. Die dritte Gruppe an Fällen fokussierte ebendieses Thema eher unter der konkreten Frage nach dem Vorliegen einer zu respektierenden Selbstbestimmtheit bei selbstgefährdenden Wünschen. Schließlich ergab sich eine weitere Reihe von Problemstellungen im Kontext Sexualität bzw. dem Wunsch nach Sexualbegleitung. Die beiden Besprechungen ,harter“ Fälle, in denen es um die Fortführung lebensverlängernder Maßnahmen oder eine Krankenhausverlegung zu diesem Zweck ging, waren retrospektiv, wurden aber als äußerst wertvoll empfunden, weil die hierin gründende moralische Belastung auch nach Jahren noch die Zusammenarbeit innerhalb der Teams beeinträchtigt hatte.

$\mathrm{Zu}$ den am meisten genannten Schwierigkeiten bei der Moderation zählten neben dem Finden und Formulieren der ethischen Fragestellung sowie der Rückführung eines Gesprächs auf das ethische Kernproblem das Finden der eigenen Rolle und Position mit moderierender und steuernder, nicht aber inhaltlich mitgestaltender Funktion.

\section{Die*der Ethikbeauftragte}

Wie bei der Vorstellung der beiden o.g. Organe deutlich wurde, spielt die Funktion der*des Ethikbeauftragten eine Schlüsselrolle bei der Umsetzung des Konzepts in die Organisationsrealität. Aus diesem Grund wurde ihre Arbeit nicht lediglich für die Übergangszeit der Implementierung benötigt, sondern als unbefristete Referentenstelle konzipiert. Ihre Aufgaben umfassen die Vermittlung, Unterstützung und Fortbildung der ethischen Fallmoderator*innen, die Systematisierung und Archivierung der EFB-Protokolle, die Planung und Organisation der Sitzungen des Ethikausschusses sowie die Leitung kleinerer Arbeitsgruppen und die Aufbereitung von Informationen zwischen Ethikausschuss und EFB. Darüber hinaus sah das Stellendesign vor, dass der*die Ethikbeauftragte selbst in dringenden team- wie klientenbezogenen Akutsituationen unter einer sogenannten „Ethik-Hotline“ für beratende Gespräche erreichbar ist.

\section{„Ethik-Hotline“6 - die telefonische Einzelberatung}

Dieses Angebot ist in jenen Fällen sinnvoll, die außerhalb von Teamprozessen entstehen oder in denen aufgrund von äußeren Zwängen (z. B. Zeitdruck) die Einberufung einer moderierten ethischen Fallbesprechung nicht möglich ist. So kamen bspw. im Fall eines ratsuchenden Facharztes, der über das Vorliegen einer medizinischen Indikation für einen Schwangerschaftsabbruch in der 20. Woche entscheiden musste, viele Faktoren zusammen: unmittelbarer Entscheidungsdruck, besonderer moralischer Gewissensdruck und (prima facie) Alleinzuständigkeit bzw. das Fehlen weiterer involvierter Fachpersonen. Die Beratung des Arztes durch die Ethikbeauftragte erstreckte sich über zwei Telefonate, wobei das erste Gespräch der Aufnahme aller wesentlichen Situationsfaktoren, der Ermittlung des Handlungsspielraums auch im Rahmen der rechtlichen Situation und der Eingrenzung der konkreten mo- 
ralischen Konfliktlage diente. Da sich zeigte, dass die Konsultation eines Kollegen entscheidende weiterführende Hinweise liefern könnte, und um Zeit für eine Ordnung der weiteren Gesprächsstruktur zu gewinnen, wurde ein weiteres Telefonat am Folgetag vereinbart. Dieses konnte sich nach einer kurzen Rekapitulation der wichtigsten Situationsfaktoren auf die Abwägung der moralischen Geltungsansprüche der beteiligten Personen konzentrieren. Die Beratung endete mit dem Ergebnis, dass sowohl für die Respektierung der Autonomie als auch den Einbezug des Wohls der schwangeren Frau entscheidende Fragen zu ihrer Situation und zur Rolle des Partners offengeblieben waren und darum schnellstmöglich eine Zweitmeinung durch einen Kollegen mit gezielter Ausrichtung auf diese Fragen einzuholen sei. Die Überweisung zu diesen Zwecken wurde als zumutbar empfunden.

In diesem Zusammenhang ist zu erwähnen, dass die telefonische Beratung spezifische Eigenheiten aufweist, die je nach Kontext als Stärke oder Schwäche zu bewerten sind. Die unmittelbare Zugänglichkeit und Erreichbarkeit der Berater*innen ermöglicht eine besonders situationsnahe Konsultation, wodurch das Problem und die moralische Verunsicherung detailliert und authentisch geschildert werden können. Gleichwohl kann sich eine unmittelbare Situationsnähe, etwa direkt nach einem schwierigen Klienten- oder Angehörigengespräch oder einem aufreibenden Versorgungsversuch, auch ungünstig auf die Strukturiertheit der Situationsbeschreibung auswirken. Zweitens sind zwar auch am Telefon Nuancen, Unsicherheiten und Ambivalenzen vermittelbar - den Beratungssuchenden gelingt dies mündlich oft wesentlich besser als in einer E-Mail -, jedoch bleibt ein Telefongespräch auf akustische Signale beschränkt, was die sorgfältige Beobachtung affektiver Hinweise (bspw. über Gestik und Mimik) der Kommunikationspartner*innen erschwert (vgl. Kon und Walter 2016, S. 517). Darüber hinaus ist freilich auch an Kommunikationsdifferenzen im interkulturellen Zusammenhang oder schlicht an Verständigungsprobleme durch verschiedene Muttersprachen zu denken. Ein bewusster Umgang mit den Limitationen des telefonischen Beratungsgesprächs kann dazu verhelfen, diesen Problemen konstruktiv zu begegnen. So kann die Interaktion gegebenenfalls auf audiovisuelle Medien (Videokonferenz) verlagert oder, wie im oben geschilderten Beispiel, in verschiedene Einzelgespräche aufgeteilt bzw. auf einen späteren Zeitpunkt verlegt werden.

Zudem erwies es sich als sinnvoll, auch im Rahmen des Telefongesprächs die grundlegende Struktur einer ethischen Fallbesprechung anzulegen, bestehend aus der Analyse von Situationsfaktoren einschließlich Ermittlung betroffener Personen (,Stakeholder"), gefolgt von einer Analyse der Wertgesichtspunkte und schließlich der Entscheidungsfindung. Mit diesem Grundgerüst vor Augen lässt sich in anderen Fällen, z.B. mit größerem Betroffenenkreis, auch ermitteln, ob das Problem eine klassische ethische Fallbesprechung mit mehreren Personen erfordert und der Zweck des Telefonats in die Vorbereitung einer solchen umzuwidmen ist. Ähnliche Vorgehensweisen sind in Eskalationsmodellen wie METAP (Albisser Schleger et al. 2012) von vornherein angelegt. 


\section{Die intermediäre Position des*der Ethikbeauftragten}

Ähnlich wie bei der Implementierung des Ethikausschusses stellte sich auch bei dem*der Ethikbeauftragten die spannungsreiche Frage nach ihrer Ansiedlung im Hierarchiensystem. „Ethikberatung ist in ihren Inhalten und in der Gestaltung des vereinbarten Vorgehens nicht weisungsgebunden. Ethikberatung ist aber Teil der Organisation und handelt folglich in einem strukturellen Kontext" (AEM 2010, S. 150). Eine zu nah an der obersten Organisationsleitung positionierte Stellung ist im Sinne ihrer Glaubwürdigkeit und Unabhängigkeit zu vermeiden (vgl. ZEKO 2006, S. A1705). Entsprechenden Vorbehalten sah sich die Ethikbeauftragte in der Anfangsphase durch ihre implementierungsbedingte Kopplung an die Stabsstelle „Unternehmensentwicklung“ gegenüber. Zugleich war zu bedenken, dass sie sich weder einem der Fachbereiche noch einer Region zuordnen lässt, sondern eine übergreifende Perspektive einnimmt. In dieser Hinsicht erkannten die Projektverantwortlichen eine Strukturähnlichkeit mit dem Bereich Theologie, weshalb die Stelle schließlich in das erweiterte Stiftungsmanagement „Theologie und Ethik“ gerückt ist.

Die Positionierungsfrage ist aber auch in einer anderen Hinsicht herausfordernd: Im Zusammenspiel von organisations- und leistungsbezogener Führungslinie ergibt sich natürlicherweise eine Art „Lagerbildung“ aus ökonomischer, juristischer, managerialer Expertise auf der einen Seite und den ausdifferenzierten Expertensystemen der Medizin, Pflege, Sozialen Arbeit, Pädagogik usw. „mit einer eigenständigen Wissensbasis, spezifischen Fachsprachen, professionellen Communities (im Sinne von Fachgesellschaften und Netzwerken), Interessenvertretungen, Publikationsorganen“ (Lobnig und Grossmann 2013, S. 7). Dabei fühlen sich die Fachleute „oft mehr ihrer Professionalität, also den Inhalten der Arbeit und den darauf bezogenen fachlichen Standards, Werten, Erfolgskriterien, Karrieremustern verpflichtet, als der Organisation" (Lobnig und Grossmann 2013, S. 7). Insofern die ethische Fallbesprechung ein Instrument der Unternehmenssteuerung ist, wird sie nicht nur vielfach als „von außen stammende“ Zusatzbelastung zur eigentlichen professionellen Arbeit gesehen, sondern sogar als Konkurrenz zu bspw. Modellen der Fallbesprechung aus den eigenen Fachrichtungen. Dies betrifft insbesondere Berufsfelder mit eigenen Modellen der Falldiskussion, regelmäßigen Teambesprechungen und Supervisionsangeboten, wie Sozialpsychiatrie und Pädagogik. Soll das Ethikmanagement also nicht nur akzeptiert, sondern aktiv „bespielt" werden, muss es der*dem Ethikbeauftragten gelingen, das Thema Ethik aus dem wahrgenommenen System „Organisation“ in den Kontext „Fachlichkeit“ zu überführen. Andernfalls droht ihr die Gefahr, als Produktmanagerin für das Produkt „Ethik“ wahrgenommen zu werden. Dies kann aufgrund der großen Eigenständigkeit der einzelnen Fachrichtungen nicht über ein zentrales Konzept, sondern nur auf individualisierte Weise und mit dementsprechend größerem Arbeitsaufwand erfolgen. Das vereinzelte „Mitlaufen“ im Arbeitsalltag von Einrichtungen hat sich für die Ethikbeauftragte als unabdingbarer Weg der ersten Kontaktaufnahme erwiesen. Dies bestätigte auch der Blick auf vergleichbare Projekte wie die „Klinische Ethik“ an den Universitären Psychiatrischen Kliniken/Universitätsspital Basel (vgl. Reiter-Theil 2016), innerhalb derer Vor-Ort- 
Besuche mit dem Angebot eines ethischen Feedbacks als gut akzeptiert, praktikabel und niedrigschwellig bewertet wurden (vgl. Reiter-Theil 2016, S. 46).

Die verschiedenartigen Herangehensweisen an die einzelnen Fachbereiche im übergreifenden Vergleich zu analysieren, wäre für multidisziplinäre, dezentrale Einrichtungen wie die BruderhausDiakonie sicher ein Gewinn, müsste aber aufgrund der Schwerpunktlegung dieses Beitrags an anderer Stelle erfolgen.

\section{Erste Beobachtungen und Ausblick}

Ziel des vorliegenden Berichts war es, exemplarisch zu zeigen, mit welchen Überlegungen die Konzeption und Implementierung eines mehrgliedrigen Ethikmanagement-Modells in dezentral organisierten Einrichtungen des Gesundheitswesens verbunden ist. Dass im Zuge dieses Unterfangens bisweilen über die Grenzen eines Standardmodells hinausgedacht werden muss, ist eine schlichte Konsequenz aus der von Neitzke treffend pointierten Tatsache: „Jede Einrichtung im Gesundheitswesen muss die ihr angemessene und zu ihr passende Institutionalisierungsform von Ethik finden und wählen“ (Neitzke 2008, S. 58).

Die Schlüsse aus der Zusammenarbeit zwischen BruderhausDiakonie und IZEW können vorerst nur auf einen ersten Abgleich mit der ideellen Wirkweise aus dem Konzeptpapier und ersten Praxiserfahrungen mit dem noch jungen Modell zurückgreifen.

\section{Problematische Präferenzen bei der Moderatorensuche}

In einem ersten Zwischenfazit lässt sich die anfangs vermutete Tendenz bestätigen, bei Bedarf nach ethischer Beratung oder Fallbesprechung die räumlich nächstgelegenen oder dem eigenen Geschäftsbereich zugehörigen Moderator*innen zu Rate zu ziehen. Die dafür angegebenen Gründe sind vielfältig und greifen doch ineinander. Zum einen gilt es im Sinne der Ressourcensparsamkeit als gute Praxis, die kürzesten Anfahrtswege und den geringsten Kommunikationsaufwand zu priorisieren.

Zum anderen scheint vielfach eine Fehleinschätzung der Moderationsrolle und der für sie erforderlichen Kompetenzen zu bestehen. Sehr hartnäckig hält sich der Eindruck, dass die moderierende Person im vorliegenden Fall auch substanziell berät und dass Fachkompetenz hierfür einen entscheidenden Vorteil darstellt. So ist bspw. Mitarbeitenden aus der Altenpflege nicht ersichtlich, warum ihnen bei Überlegungen zu angemessenen Pflegeinterventionen am Lebensende ein*e Ethikmoderator*in aus dem Bereich Kinder- und Jugendhilfe hilfreich sein könnte. Dass es bei der Moderation ethischer Fallbesprechungen um ethische und Gesprächsführungskompetenz geht (vgl. Vollmann 2017, S. 198) und Moderator*innen nicht die Rolle fachlicher Supervisor*innen, sondern die ,des ausgleichenden Leiters des Gruppenprozesses“ (vgl. Vollmann 2017, S. 200) zukommt, kann darum in Informationsgesprächen nicht stark genug betont werden.

Eine weitere Rolle bei dem Wunsch, ethische Konflikte in der eigenen Einrichtung oder Geschäftsstelle ,nicht nach außen zu tragen“, spielt die Sorge, auf mangelndes Verständnis und moralische Verurteilung zu treffen. Innerhalb des eigenen Fach- 
bereichs ist die grundsätzliche Haltung hingegen eher von Solidarität geprägt (vgl. auch Sauer 2015, S. 117). Diese Vorbehalte sind ernst zu nehmen und lassen sich nicht allein durch Vertraulichkeitsvorschriften entkräften, sondern langfristig nur durch die Entmystifizierung von Ethik als „moralischem Tribunal“ durch gezielte Aufklärung und erfolgreiche Fallbesprechungen.

Festzuhalten bleibt, dass sich der Kollegen- oder Mitarbeiterstatus nicht mit der erforderlichen Unparteilichkeit und Unabhängigkeit der Moderationsrolle verträgt, deren zentrale Aufgabe es ist, verfahrensethische Rahmenbedingungen des (hierarchie-)freien und gleichberechtigten Diskurses zu gewährleisten (vgl. BaumannHölzle 2009, S. 222). Dabei ist auch zu bedenken, dass eine starke professionelle Nähe zum vorliegenden Handlungsfeld dazu verleiten kann, in der Berufsrolle zu verbleiben, sich für die fachliche Qualität der Entscheidung mitverantwortlich zu fühlen, fachliche mit ethischen Fragen zu verwechseln (vgl. auch Vollmann 2017, S. 200) oder sich an kleinteiligen Nebendiskursen zu beteiligen, anstatt diese in das ethisch-moralische Gesamtbild zurückzuführen. Aus diesem Grund bleibt der Einsatz von Moderator*innen vorzugswürdig, die nicht aus der betreffenden Einrichtung stammen und nicht primär im vorliegenden Themengebiet tätig sind.

Es sind solche organisations- bzw. verfahrensethischen Zusammenhänge, für die es neben der materialethischen Grundkenntnis ein allgemeines Bewusstsein zu schaffen gilt. Damit dies gelingen kann, ist abermals die Rolle und Sichtbarkeit der*des Ethikbeauftragten hervorzuheben, welche sich nicht mit einer Telefonnummer im internen Adressbuch erschöpft, sondern vor allem durch regelhafte Vor-Ort-Besuche, Inhouse-Workshops, aktive und individualisierte Gesprächsangebote in Einrichtungen aller Regionen gewonnen werden kann.

\section{Hierarchie und Partizipation}

Unabhängig von der Präferierung ,hauseigener“ Moderator*innen ist zu konstatieren, dass die meisten, eventuell sogar alle ${ }^{4}$ Anträge von Führungskräften initiiert wurden. Dieser Umstand deutet darauf hin, dass für die Mitarbeiterschaft, die Patient*innen und Angehörigen entweder ein geringer Bekanntheitsgrad des Angebots oder hierarchiebedingte Hemmnisse bei seiner Inanspruchnahme bestehen. Vereinzelte Gespräche mit Mitarbeitenden und Führungskräften deuten darauf hin, dass eine untergeordnete Stellung im organisationalen Weisungsgefüge mit einer geringen Wahrnehmung der aktiven Partizipationsmöglichkeiten an Angeboten der Ethikberatung einhergeht. Im Hintergrund stehen Vorbehalte, das eigene Gefühl der moralischen Verunsicherung auf die Ebene eines offiziellen Gesprächs bzw. Termins mit Beteiligung Dritter zu heben und dadurch Konflikte mit Vorgesetzten zu riskieren. Vieles spricht dafür, dass Hemmnisse dieser Art auch für Patient*innen und Angehörige zum Tragen kommen, die sich in Abhängigkeitsverhältnissen mit versorgenden Personen bzw. Teams befinden (vgl. Gacki-Smith und Gordon 2005). Nicht zuletzt sind auch jene Dynamiken zu beachten, die unter dem Stichwort der ,epistemischen Ungerechtigkeit“ (Fricker 2005) verhandelt werden: das Zurückbleiben relevanter

\footnotetext{
${ }^{4}$ In wenigen Fällen ist nicht mehr nachvollziehbar, von wem die Initiative ausging.
} 
Stakeholder hinter einem für Mitgestaltungsmacht entscheidenden Wissens- oder Kenntnisstand (vgl. Ho und Unger 2015).

Auf Barrieren wie diese lässt sich nicht immer unmittelbar zugreifen. Gute $\mathrm{Zu}$ gänglichkeit von Informationsmaterial für alle Zielgruppen (hierzu gehören bspw. Flyer in Leichter Sprache und gute Auffindbarkeit der Ethikarbeit auf der Homepage) ist zwar eine notwendige aber nicht zwingend hinreichende Voraussetzung für Partizipationsgerechtigkeit. Entscheidende Hindernisse können auch im Verfahren der Antragstellung liegen. Dieses kann zu kompliziert gestaltet sein oder selbst eine hierarchische Hürde enthalten. Seit kurzem ist bspw. festzustellen, dass anstelle der offiziellen Antragsroutine der BruderhausDiakonie, die eine schriftliche Antragstellung mit Entscheid durch eine Führungskraft vorsieht, vermehrt die Ethikbeauftragte angerufen und um Abwicklung der Formalia einschließlich Moderator*innensuche gebeten wird. Darum ist zu prüfen, ob die ursprüngliche Praxis flexibler gestaltet werden kann, ohne die Entscheidungsfunktion der Führungskräfte zu schwächen.

Ein weiterer, ungleich aufwändigerer Weg ist der aktive Einbezug der Mitarbeitenden, Patient*innen und Angehörigen in Gesprächskreise und Fachtage sowie die Entwicklung kleinerer, zielgruppengerechter Diskursformate. So zeigte sich bspw. bei der Gründung des Frankfurter Netzwerks „Ethik in der Altenpflege“, dass der ursprünglich nur als ergänzende Maßnahme geplante „Gesprächskreis Ethik“ von der Mitarbeiterschaft so gut angenommen wurde, dass er ,ein wesentliches Element der Netwerkstruktur des Projekts“ (Sauer 2015, S. 118) wurde. Für die aktive Kontaktaufnahme erscheint es zudem sinnvoll, die bestehenden Kommunikationsstrukturen, bspw. Beiräte, zu nutzen. Denkbar wäre darüber hinaus die regelmäßige Einladung von Mitarbeitenden, Patient*innen und Angehörigen oder deren Vertreter*innen zu einem offenen Teil einer Ethikausschusssitzung.

Eine systematische Evaluation des Ethikmanagement-Modells steht indessen noch aus. Sie kann sowohl für die wissenschaftliche Ethik als auch für die interne Organisationsentwicklung fruchtbare Erkenntnisse hervorbringen, etwa über die Fragen, in welchen Geschäftsfeldern welche Instrumente besser oder schlechter Fuß fassen, ob das Ethikmodell alle benötigten Formen ethischer Reflexion abdeckt oder ob sich die Arbeit von Ausschuss und EFB tatsächlich zentral verknüpfen lässt.

Es ist nun, zwei Jahre nach Abschluss der Implementierungsphase, klar zu erkennen, dass die Anzahl an EFB mit der regionalen Dichte an Ethikmoderator*innen sowie dem regionalen Bekanntheitsgrad der*des Ethikbeauftragten korreliert. Vermeintliche Details wie die Freistellung der Ethikmoderator*innen für ihre Arbeit oder der Stellenumfang der Ethikbeauftragten sind darum keine „Kleinigkeiten“, sondern Faktoren, die entscheidend zum Gelingen eines Ethikberatungsmodells beitragen können und bereits in der Konzeption mitgedacht werden müssen.

Ein für alle Seiten wünschenswertes Fernziel eines solchen Unterfangens wäre die gezielte Entwicklung von Qualitätsmerkmalen. Auch diese ist aber in jedem Fall auf die Kapazitäten einer zentralen Person wie der Ethikbeauftragten (-koordinator*in, -referent*in) angewiesen, mit wissenschaftlichen Kooperationspartnern zusammenzuarbeiten und mit gebotener Sorgfalt Daten zu erheben und aufzubereiten.

Danksagung Wir danken den anonymen Gutachter*innen für ihre gewinnbringenden Hinweise, welche zu wertvollen Ergänzungen und Differenzierungen geführt haben. 
Funding Open Access funding enabled and organized by Projekt DEAL.

Open Access Dieser Artikel wird unter der Creative Commons Namensnennung 4.0 International Lizenz veröffentlicht, welche die Nutzung, Vervielfältigung, Bearbeitung, Verbreitung und Wiedergabe in jeglichem Medium und Format erlaubt, sofern Sie den/die ursprünglichen Autor(en) und die Quelle ordnungsgemäß nennen, einen Link zur Creative Commons Lizenz beifügen und angeben, ob Änderungen vorgenommen wurden.

Die in diesem Artikel enthaltenen Bilder und sonstiges Drittmaterial unterliegen ebenfalls der genannten Creative Commons Lizenz, sofern sich aus der Abbildungslegende nichts anderes ergibt. Sofern das betreffende Material nicht unter der genannten Creative Commons Lizenz steht und die betreffende Handlung nicht nach gesetzlichen Vorschriften erlaubt ist, ist für die oben aufgeführten Weiterverwendungen des Materials die Einwilligung des jeweiligen Rechteinhabers einzuholen.

Weitere Details zur Lizenz entnehmen Sie bitte der Lizenzinformation auf http://creativecommons.org/ licenses/by/4.0/deed.de.

\section{Einhaltung ethischer Richtlinien}

Interessenkonflikt C. Burmeister, A. Iller, R. Ranisch, C. Brand, T. Staib und U. Müller geben an, dass kein Interessenkonflikt besteht.

Ethische Standards Für diesen Beitrag wurden von den Autoren keine Studien an Menschen oder Tieren durchgeführt. Für die aufgeführten Studien gelten die jeweils dort angegebenen ethischen Richtlinien.

\section{Literatur}

AEM (2010) Standards für Ethikberatung in Einrichtungen des Gesundheitswesens. Ethik Med 22:149-153 Albisser Schleger H, Merz M, Meyer-Zehnder B, Reiter-Theil S (2012) Klinische Ethik - METAP. Leitlinie für Entscheidungen am Krankenbett. Springer, Berlin

Baumann-Hölzle R (2009) 7 Schritte Dialog - Exemplarische Vertiefung der Methodik einer Fallbesprechung. In: Arn C, Baumann-Hölzle R (Hrsg) Ethiktransfer in Organisationen. Handbuch Ethik im Gesundheitswesen, Bd. 03. Schwabe, Basel, S 9-14

BruderhausDiakonie (2020) Das Leitbild der BruderhausDiakonie. https://www.bruderhausdiakonie.de/ fileadmin/redaktion/Allgemein_BruderhausDiakonie/Unternehmen/Ueber_die_BruderhausDiakonie/ Leitbild/2020_Leitbild_ND_web.pdf. Zugegriffen: 20. Apr. 2021

Fricker M (2005) Epistemic injustice: power and the ethics of knowing. Oxford University Press, London

Gacki-Smith J, Gordon E (2005) Residents' access to ethics consultations: knowledge, use, and perceptions. Acad Med 89(2):168-175

Ho A, Unger D (2015) Power hierarchy and epistemic injustice in clinical ethics consultation. Am J Bioeth $15(1): 40-42$

Kon AA, Walter RJ (2016) Health care ethics consultation via telemedicine: linking expert clinical ethicists and local consultants. AMA J Ethics 18(5):514-520

Lobnig H, Grossmann R (2013) Organisationsentwicklung im Krankenhaus. MWV, Berlin

Maaser W (2013) Sozialarbeiterische Profession im Spannungsfeld von normativem Selbstverständnis und sozialstaatlicher Beauftragung. Ethik J 1(1):1-18

Neitzke G (2008) Aufgaben und Modelle klinischer Ethikberatung. In: Dörries A, Neitzke G, Simon A, Vollmann J (Hrsg) Klinische Ethikberatung. Kohlhammer, Stuttgart, S 58-75

Rabe M (2017) Ethik in der Pflegeausbildung. Hogrefe, Bern

Ranisch R, Brand C (2016) Clinical ethics consultation and the challenge to implement what is right. Am J Bioeth 16(9):38-39

Reiter-Theil S (2016) Initiating and maintaining clinical ethics support in psychiatry. Ten tasks and challenges - and how to meet them. Clin Ethics 11(2/3):45-53

Sauer T (2015) Das Frankfurter Netzwerk ,Ethik in der Altenpflege“. In: Coors M, Simon A, Stiemerling M (Hrsg) Ethikberatung in Pflege und ambulanter Versorgung. Jacobs, Lage, S 114-126 
Steinkamp N, Gordijn B (2010) Ethik in der Klinik - Ein Arbeitsbuch. Zwischen Leitbild und Stationsalltag. Luchterhand, Köln

Thiersch H (2015) Moral und Soziale Arbeit. In: Otto HU, Thiersch H (Hrsg) Handbuch Soziale Arbeit: Grundlagen der Sozialarbeit und Sozialpädagogik. Reinhardt, München, Basel, S 1058-1069

Vollmann J (2002) Klinische Ethikkomitees: Zur aktuellen Entwicklung in deutschen Krankenhäusern. In: Kolb S (Hrsg) Medizin und Gewissen. Wenn Würde ein Wert würde. Menschenrechte, Technologiefolgen, Gesundheitspolitik. Mabuse, Frankfurt, S 276-287

Vollmann J (2008) Implementierung einer klinischen Ethikberatung. Prozess der Implementierung. In: Dörries A, Neitzke G, Simon A, Vollmann J (Hrsg) Klinische Ethikberatung. Kohlhammer, Stuttgart, S 116-128

Vollmann J (2017) Klinische Ethikkomitees und Klinische Ethikberatung. In: Vollmann J (Hrsg) Ethik in der Psychiatrie: Ein Praxisbuch. Psychiatrie Verlag, Hamburg, S 178-187

ZEKO (2006) Stellungnahme: Ethikberatung in der klinischen Medizin. Dtsch Arztebl 103(24):A 1703-A 1707 